\title{
Prognostic significance of apoptotic cell death in bladder cancer: a tissue microarray study on 179 urothelial carcinomas from cystectomy specimens
}

\author{
Eva Karamitopoulou*, Cyrill A. Rentsch†, Regula Markwalder $\$$, \\ Claudio Vallanş, George N. Thalmann† and Thomas Brunner: \\ *2nd Department of Pathology, University of Athens, Attikon University Hospital, Athens, Greece, †Department \\ of Urology, Inselspital, Berne, $\$$ Institute of Pathology, University of Berne, and §FACS Facility, Department of \\ Clinical Research, University of Berne, Berne, Switzerland
}

\begin{abstract}
Summary
Aims: To assess the prognostic significance of apoptosis related markers in bladder cancer.

Methods: A tissue microarray containing 179 bladder carcinomas from cystectomy specimens was analysed immunohistochemically for active caspase-3, single-stranded DNA (ssDNA), p53, Bcl-2, Bax, and COX-2, in correlation to clinicopathological factors.

Results: Active caspase-3, ssDNA, p53, Bax and COX-2 were more frequently observed among high grade and higher stage ( $\geq T 2$ ) carcinomas compared with low grade and lower stage (T1) tumours. On the contrary, Bcl-2 was more frequently detected in $\mathrm{T} 1$ than in $\geq \mathrm{T} 2$ carcinomas. Active caspase-3 correlated with a better survival of the patients. Conclusions: The decreased detection of active caspase-3 and ssDNA and the increased presence of Bcl-2 in T1 carcinomas suggest that alterations in interrelated apoptosis markers may play an important role in the progression of urothelial carcinoma from a superficially infiltrating to a muscle invading tumour and would help to better characterise a subpopulation of $\mathrm{T} 1$ carcinomas that could profit from early cystectomy or more aggressive adjuvant chemotherapy. Active caspase-3 might be an important prognostic factor in bladder cancer.
\end{abstract}

Key words: Apoptosis, active caspase-3, ssDNA, bladder cancer.

\section{INTRODUCTION}

Genetic changes affecting proliferation and apoptosis contribute to the development of urothelial carcinoma. ${ }^{1-3}$ Alterations in gene products regulating apoptosis in bladder cancer cells like p53, Bcl-2 and Bax are associated with decreased apoptotic cell death and increased cell survival. ${ }^{4.5}$ The morphological changes of apoptosis are mediated by a group of cysteine proteases, the caspases. Caspases play an essential role in the initiation, regulation and execution of apoptosis. ${ }^{6-8}$ Particularly, caspase-3 is required for cleavage of a large number of proteins and of nuclear collapse during apoptosis." Similarly, DNA fragmentation is observed in all nucleated cells. The immunohistochemical detection of active caspase-3 and single-stranded DNA (ssDNA) therefore could represent a valuable tool for the identification of apoptotic cells in tissue sections. ${ }^{10,11}$

In the present study we employed immunohistochemistry for active caspase-3 and ssDNA for the detection of apoptosis in 179 bladder carcinomas from cystectomy specimens combined in a tissue microarray (TMA). The results were compared with the expression of proteins involved in the modulation of apoptosis, such as p53, Bcl-2, Bax, and COX-2, and were correlated to the stage of the disease, and the clinical outcome.

\section{MATERIALS AND METHODS}

Patients and specimens

Formalin-fixed, paraffin-embedded radical cystectomy specimens from 179 patients (129 males and 50 females; mean age 67 years, range 3586 years) with invasive urothelial carcinoma of the bladder, and 42 normal controls were used. According to the sixth edition of the TNM classification of malignant tumours, the 179 tumours included 17 pT1, 26 pT2, 103 pT3 and 33 pT4 cases. $^{12}$ Lymph node metastases were present in 39 patients $(22 \%)$. According to the 2004 World Health Organization (WHO) and International Society of Urological Pathology (ISUP) classification, the majority of the tumours (i.e., 169) were WHO high grade while 10 tumours were low grade. $^{13}$

Construction of tissue microarray (TMA)

TMA was built as previously described. ${ }^{4}$ Briefly, one core tissue biopsy with a diameter of $0.6 \mathrm{~mm}$ was taken from a representative region of individual paraffin embedded bladder carcinomas (donor blocks) and placed into a new recipient paraffin block using a semi-automated tissue arraying device. The presence of tumour tissue on the TMA was verified on a haematoxylin and eosin (H\&E) stained slide. Two to three tissue cores of each tumour were available for biomarker analysis. Five $\mu \mathrm{m}$ sections were cut using an adhesive-coated slide system (Instrumedics, USA) and examined by immunohistochemistry. The number of samples varied slightly between the individual markers because of variability in the number of interpretable specimens on TMA sections.

\section{Assessment of behaviour}

Medical charts were available from 175 patients $(98 \% ; 126$ males and 49 females). Of these, $81(46.28 \%)$ died of the disease, and $59(33.7 \%)$ were alive without recurrent/metastatic disease. An additional 35 patients $(20 \%)$ died of other causes. Mean follow-up was 57 months (median 40; range 1 200; SD 49.8) 


\section{Immunohistochemistry}

TMA sections were deparaffinised, rehydrated and pretreated for $10 \mathrm{~min}$ in $10 \mathrm{mM}$ citrate $\mathrm{pH} 6.0$ in a pressure cooker. They were then incubated with the following primary antibodies: anti-active caspase-3 (1:100; Bio-concept, USA), anti-ssDNA (clone F7-26, $10 \mu \mathrm{g} / \mathrm{mL}$, Apostain; Alexis Biochemicals, USA), p53 (clone DO-7, 1:200; Novocastra, UK), Bcl-2 (clone 124, 1:200; DakoCytomation, Denmark), Bax (clone 2D2, 1:100; Neomarkers, USA) and COX-2 (clone CX294, 1:25; DakoCytomation) according to the manufacturers' protocols. In negative controls the primary antibody was replaced with an irrelevant, isotype-matched antibody. As positive control, sections from a TMA with many different samples of normal tissue were stained in parallel.

\section{Scoring of immunohistochemistry}

The scoring was performed by a staff pathologist with expertise in genitourinary pathology who was blinded to clinical outcome and stage of the tumours.

For active caspase- 3 and ssDNA, positive tumour cells were identified by high power field, and assigned to one of four categories: (a) negative, (b) $14 \%$, (c) $510 \%$, and (d) $>10 \%$ positive cells.

For p53, only nuclear staining was considered and a cut off of $10 \%$ was required to define positivity. High p53 immunoreactivity was defined as positivity in $>20 \%$ of the tumour cell nuclei.

For Bcl-2, COX-2 and Bax, the intensity of immunostaining was visually scored and stratified into four groups: (a) negative, (b) weak, (c) moderate, and (d) strong. At least moderate staining in $>10 \%$ of tumour cells was requested to define positivity.

\section{Statistical analysis}

Kaplan Meier plots were used to estimate overall survival from cystectomy to the date of death from any cause or to the date of relapse. Differences among subgroups were assessed using the log-rank test. Significance level was set at $p<0.05$.

Kendall's Tau-b was used to measure the correlation coefficient of the expression of immunohistochemical markers to the histological parameters and stage of tumours. The levels of significance were determined by a twotailed test.

\section{RESULTS}

Fig. 1 shows typical examples of active caspase-3 (Fig. 1A,B) and ssDNA staining (Fig. 1C,D), whereas Fig. 2 illustrates p53 (Fig. 2A), Bcl-2 (Fig. 2B), Bax (Fig. 2C) and COX-2 (Fig. 2D) staining in urothelial carcinoma.

Active caspase- 3 was detected in the cytoplasm of cells showing typical apoptotic morphology (Fig. 1A,B). No positive staining was observed in areas of necrosis or in normal urothelium. Positive reaction for caspase-3 was found in $45.34 \%$ of tumours (Table 1).

Active caspase- 3 positive cells were significantly more frequent in high grade compared with low grade carcinomas ( $p=0.024$; Fig. 3A). In addition, active caspase-3 immunoreactivity was more frequently present in pT2 or higher stage tumours as opposed to pT1 carcinomas $(p=0.044)$, and was more often detected in nodal positive $(62.8 \%)$ compared with nodal negative carcinomas $(40.5 \%)$ (Table 2); however, this difference was not statistically significant.

ssDNA immunoreactivity was present in $39.4 \%$ of tumours. Most positive cells exhibited a nuclear staining.
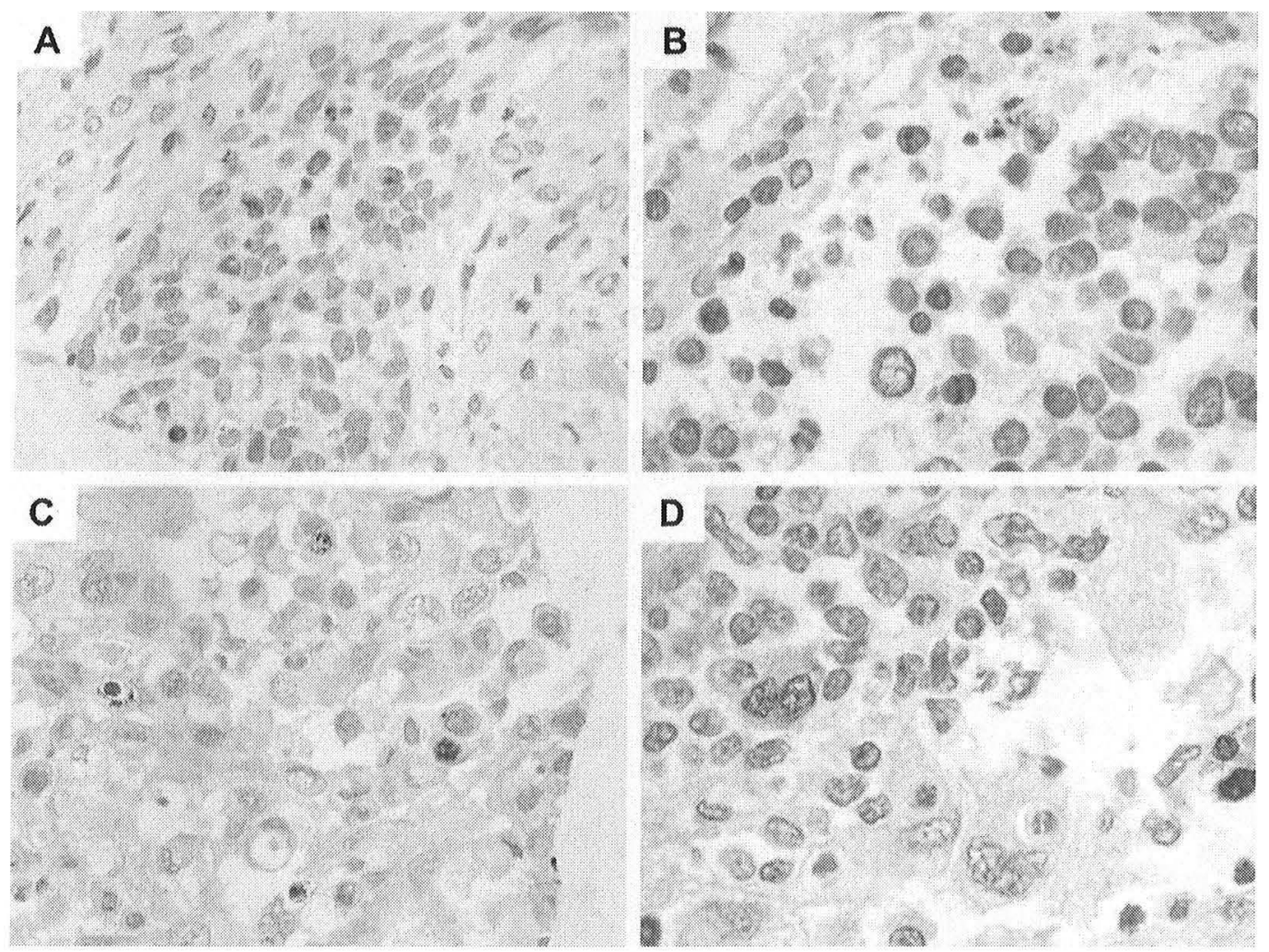

Fig. 1 Immunohistochemical detection of active caspase-3 and ssDNA in bladder cancer. (A,B) Specific staining of active caspase-3 and (C,D) ssDNA in cases of bladder cancer. (B,D) Enlarged details of cytoplasmic active caspase-3 and nuclear ssDNA positivity in urothelial carcinoma. Typical examples are shown. 



Fig. 2 (A) Nuclear p53, (B) cytoplasmic Bcl-2, (C) Bax and (D) COX-2 immunoreactivity in cases of urothelial carcinoma of the bladder. Typical examples are shown.

Table 1 Immunohistochemical findings in correlation with the T-stage of bladder cancer

\begin{tabular}{lcccccc}
\hline Pathological T-stage & Caspase-3 & ssDNA & p53 & Bcl-2 & Bax & COX-2 \\
\hline T1 & $3 / 13(23 \%)$ & $3 / 13(23 \%)$ & $4 / 13(30.7 \%)$ & $3 / 13(23 \%)$ & $3 / 13(23 \%)$ & $0 / 12(0 \%)$ \\
T2 & $13 / 25(52 \%)$ & $14 / 24(58.3 \%)$ & $13 / 25(52 \%)$ & $2 / 24(8.3 \%)$ & $16 / 25(64 \%)$ & $8 / 25(32 \%)$ \\
T3 & $42 / 94(44.7 \%)$ & $40 / 99(40.4 \%)$ & $66 / 100(66 \%)$ & $6 / 94(6.4 \%)$ & $51 / 93(54.8 \%)$ & $44 / 99(44.4 \%)$ \\
T4 & $15 / 29(51.7 \%)$ & $8 / 29(27.6 \%)$ & $20 / 29(68.9 \%)$ & $1 / 29(3.4 \%)$ & $10 / 28(35.7 \%)$ & $8 / 27(29.6 \%)$ \\
ZT2 & $70 / 148(47.3 \%)$ & $62 / 152(41 \%)$ & $99 / 154(64 \%)$ & $9 / 147(9.5 \%)$ & $76 / 147(51.7 \%)$ & $61 / 151(40.4 \%)$ \\
Overall & $73 / 161(45.34 \%)$ & $65 / 165(39.4 \%)$ & $103 / 167(61.7 \%)$ & $12 / 160(7.5 \%)$ & $80 / 159(50.3 \%)$ & $60 / 163(36.8 \%)$ \\
$p$ value & $\mathbf{0 . 0 4 4}$ & 0.979 & $\mathbf{0 . 0 4 9}$ & 0.978 & 0.254 & 0.059 \\
\hline
\end{tabular}

The number of samples varied between the individual markers because of differences in the number of the interpretable tumour specimens on tissue microarray sections.

A

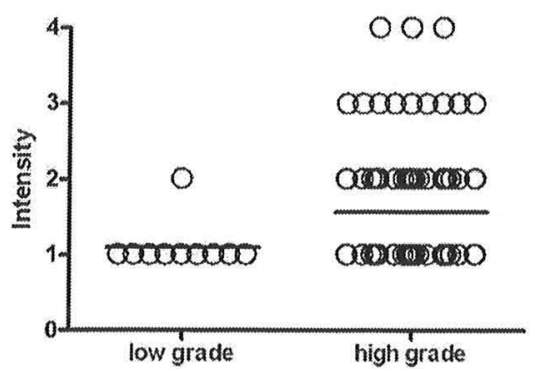

B

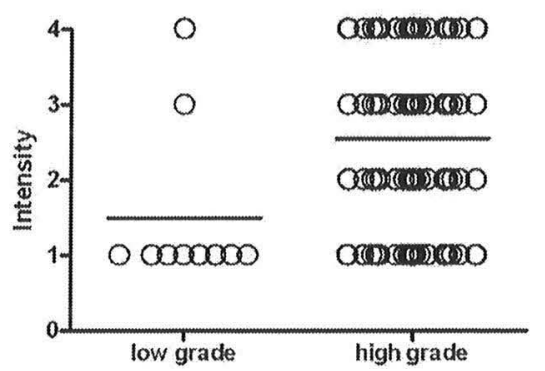

C

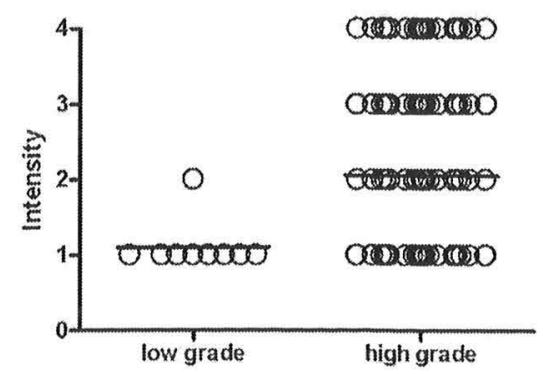

Fig. 3 Distribution of the scoring for (A) active caspase-3, (B) p53 and (C) COX-2 between low grade and high grade bladder cancer. Each circle represents the score of one patient. 
In some cells also a cytoplasmic staining was noted, most likely reflecting the release of ssDNA into the cytoplasm (Fig. 1C,D). Areas of tumour necrosis and normal urothelium remained immunonegative. Muscle invading tumours (i.e., $\geq \mathrm{pT} 2$ ) showed an increased number of ssDNA positive cells $(41 \%)$ compared with pT 1 carcinomas $(23 \% ; p=0.979)$.

Positive nuclear p53 staining was observed in $61.7 \%$ of tumours and correlated significantly with the T-stage, being found in $30.7 \%$ of pT1 compared with $64 \%$ of $\geq \mathrm{pT} 2$ carcinomas ( $p=0.049$; Table 1; Fig. 2A). Moreover, p53 immunoreactivity was significantly more frequent in high grade as opposed to low grade carcinomas $(p=0.018$; Fig. 3B).

Positive cytoplasmic immunoreactivity for Bcl-2, COX-2 and Bax was seen in $7.5 \%, 36.8 \%$ and $50.3 \%$ of tumours, respectively (Fig. 2A-D). COX-2 was significantly more frequently found in high grade as opposed to low grade neoplasms $(p=0.019$; Fig. $3 \mathrm{C})$. COX-2 and Bax were more often found in higher stage neoplasms exhibiting immunopositive reaction in $0 \%$ and $23 \%$ of pTl compared with $40.4 \%$ and $51.7 \%$ of $\geq \mathrm{pT} 2$ carcinomas, respectively. In contrast, Bcl-2 immunoreactivity was more commonly found in pT1 tumours $(23 \%)$ than in the muscle invading tumours $(9.5 \%$; Table 1). COX-2 expression correlated significantly with the presence of active caspase-3 $(p=0.03)$.

\section{Correlation with survival}

The presence of active caspase- 3 correlated with a better survival in this series, since patients with active caspase-3 positive tumours survived 1 year longer than patients with active caspase- 3 negative carcinomas ( $p=0.0260$; Fig. 4A). The presence of ssDNA did not correlate with survival.

The presence of low p53 immunoreactivity (i.e., 10-20\%) correlated with a better survival in all patients as well as in patients dying of bladder cancer after cystectomy, compared with absence of (i.e., $<10 \%$ ) or high (i.e., $>20 \%$ ) p53 immunoreactivity ( $p=0.0124$; Fig. 4B, C).

No correlation between Bcl-2, Bax and/or COX-2 and survival of the patients was noted.

\section{DISCUSSION}

While current histopathological criteria can provide important morphological information about tumour stage and grade, they have clear limits in specifying the risk of progression or response to treatment for an individual patient with urothelial carcinoma. ${ }^{15}$ Definition of individual prognostic markers is mandatory for a standardised and optimised therapy. ${ }^{16}$ Apoptotic cell death is as important as cell proliferation for tumour growth. ${ }^{17}$ Therefore, the detection of apoptosis in situ and its correlation with the expression of pro- and anti-apoptotic molecules seems to be worth further evaluation. In this study we evaluated active caspase- 3 and ssDNA for the detection of apoptosis in bladder cancer in correlation to clinicopathological factors.

Active caspase- 3 immunoreactivity correlated with unfavourable histological parameters such as high grade and advanced stage of tumours. This finding is in agreement with a previous report ${ }^{18}$ which showed that overexpression of activated caspase- 3 was associated with a high chance of disease invasiveness. Although this may appear contradictory at first glance, there are numerous reports that link deregulated cell cycle progression with induction of apoptosis. For example, the oncogene c-Myc, often overexpressed in tumour cells, promotes cell proliferation, but may also induce apoptosis in the absence of sufficient survival signals. ${ }^{19}$ Since advanced tumours frequently show impaired histological differentiation, we assume our observation in the context of an increased cellular turnover

Table 2 Immunohistochemical findings in correlation with the $\mathrm{N}$-stage of bladder cancer

\begin{tabular}{lccccc}
\hline N-stage & Caspase-3 & ssDNA & p53 & Bcl-2 & Bax \\
\hline N0 & $51 / 126(40.5 \%)$ & $51 / 130(39.2 \%)$ & $79 / 130(60.7 \%)$ & $8 / 125(6.4 \%)$ & $64 / 125(51.2 \%)$ \\
N1/2 & $22 / 35(62.8 \%)$ & $14 / 35(40 \%)$ & $24 / 37(64.8 \%)$ & $4 / 35(11.4 \%)$ & $16 / 34(47 \%)$ \\
$p$ value & 0.219 & 0.799 & 0.527 & 0.451 & $15 / 34(44.1 \%)$ \\
\hline
\end{tabular}

A

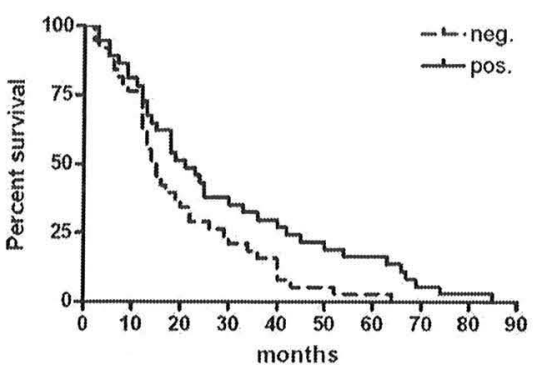

B

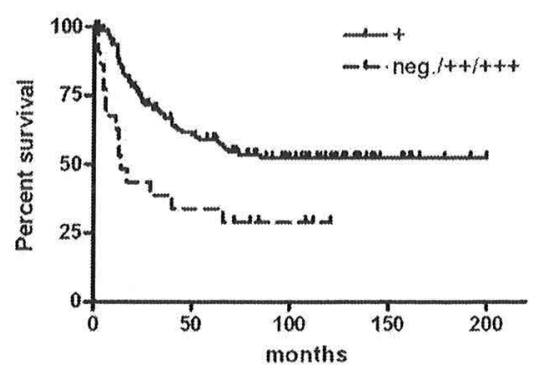

C

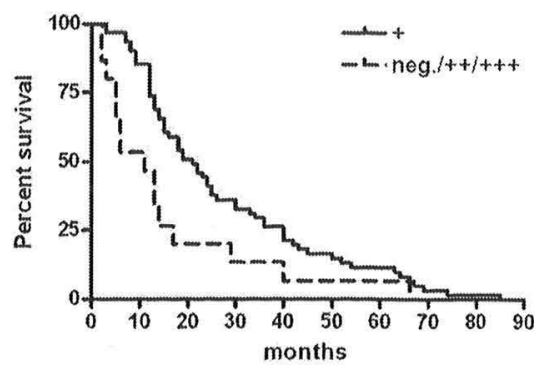

Fig. 4 Kaplan Meier plots for percent survival after radical cystectomy for patients with residual tumour at the time of operation. Ticks represent death from other cause or loss to follow up. (A) Kaplan Meier plot comparing the subgroups of patients dying of bladder cancer with active caspase-3 positive and active caspase-3 negative tumours. (B) Comparison of survival for patients with tumours presenting with slightly positive ( + ) p53 staining, and no or more positive (negative/ $++/+++$ ) staining. (C) Same as (B) for the subgroup of patients dying of bladder cancer. 
that results in a higher rate of apoptosis. However, active caspase- 3 immunoreactivity correlated significantly with a better survival of the patients $(p<0.05$; Fig. 3A). This finding indicates that active caspase- 3 might be an important prognostic factor for bladder cancer and could be attributed to an increased rate of apoptotic cell death leading to accelerated tumour involution. Moreover, it could be hypothesised that active caspase- 3 positive tumours would be more susceptible to induction of apoptotic cell death by chemotherapeutic agents compared with active caspase-3 negative, apoptosis resistant neoplasms. Our findings are partly in agreement with those of Karam and co-workers ${ }^{20}$ who also showed that altered apoptosis markers constitute an important prognostic indicator for patients with bladder cancer.

Our study also revealed that active caspase- 3 and ssDNA are not equally sensitive in detecting apoptotic cells in urothelial carcinoma. This may be attributed to different reasons. Caspase- 3 activation represents an early and central step in apoptotic pathway. In contrast, DNA degradation occurs later and is often dependent on caspase-3 activation. ${ }^{21}$ Moreover, late apoptotic cells exhibiting DNA degradation are often removed by phagocytes and are therefore more difficult to detect. This could also partly explain the fact that ssDNA positivity, in contrast to active caspase-3, did not have any influence on the survival of the patients.

p53 is a transcription factor that can induce the expression of multiple pro-apoptotic gene products, including caspase activators. ${ }^{22}$ In the present study, p53 immunoreactivity correlated significantly with increased tumour stage $(p<0.05$; Table 1$)$ and caspase- 3 positivity $(p=0.013)$. Moreover, p53 staining was associated with decreased overall survival of the patients. However, the presence of low p53 immunoreactivity (i.e., 10-20\%) correlated with a better survival in all patients as well as in patients dying of bladder cancer after cystectomy, compared with absence of (i.e., $<10 \%$ ) or high (i.e., $>20 \%$ ) p53 immunoreactivity (Fig. 3B). Absence of p53 immunoreactivity is likely the result of either degraded p53 or deletion of the p53 gene. Clearly this results in impaired functions of this tumour suppressor gene and reduced apoptosis in response to DNA damaging agents and thereby to reduced survival of the patients. In contrast, low p53 immunoreactivity could be attributed to the presence of wild-type $\mathrm{p} 53$, which may have maintained its normal tumour suppressor functions, thus triggering apoptosis and being associated with better survival of the patients. Finally, high immunoreactivity in the absence of increased survival of the patients may be associated with high expression of a mutated form of p53 incapable of activating apoptosis pathway and thereby executing the tumour suppressor functions of $\mathrm{p} 53$. Previous studies ${ }^{23,24}$ showed considerable differences concerning the prognostic significance of p53 tumour status in bladder cancer, which was attributed to the different p53 immunohistochemistry protocols used, patient selection and study design. Our findings suggest that some of the differences may be related to quantitative differences in p53 expression of the tumour cells.

The Bcl-2 family of proteins is known to play a pivotal role in the regulation of apoptosis. ${ }^{25}$ In our study, Bcl-2 was more frequently expressed in lower stage tumours that were mostly caspase- 3 and ssDNA negative. This finding is compatible with the anti-apoptotic function of $\mathrm{Bcl}-2$. On the contrary, Bax immunoreactivity was more often present in higher stage tumours, frequently showing active caspase- 3 and ssDNA positivity, supporting a proapoptotic function of this protein.

A further factor conferring resistance to apoptosis is cyclooxygenases. ${ }^{26}$ Previous studies ${ }^{27}$ showed that COX-2 correlated with local invasion, lymphovascular invasion and recurrence in bladder carcinomas. In our study COX-2 immunoreactivity was strongly associated with muscle invasion, and correlated significantly with the presence of active caspase- $3(p=0.03)$.

Our results show that apoptotic cell death, as assessed by active caspase- 3 and ssDNA immunohistochemical detection, is a common finding in bladder cancer. The decreased expression of active caspase-3 and ssDNA and the increased presence of Bcl-2 in the pT1 carcinomas suggest that alterations in interrelated apoptosis markers may play an important role in the progression of urothelial carcinoma from a superficially infiltrating to a muscle invading tumour. Since it is known that pT1 bladder carcinomas represent a heterogeneous group of tumours, this finding might help to better characterise a subpopulation of pT1 carcinomas that could profit from early cystectomy or aggressive chemotherapy. The presence of active caspase- 3 immunoreactivity correlated with better survival of the patients indicating that active caspase- 3 might be a relevant prognostic factor for patients with bladder cancer.

Address for correspondence: Professor T. Brunner, Division of Immunopathology, Institute of Pathology, University of Berne, Murtenstrasse 31, 3010 Berne, Switzerland. E-mail: tbrunner(a) pathology.unibe.ch. Dr E. Karamitopoulou, 2nd Department of Pathology, University of Athens, Attikon University Hospital, Rimini 1, 12464 Haidari, Athens, Greece. E-mail: ekaramit@med.uoa.gr

\section{References}

1. Baithun SI, Naase M, Blanes A, Diaz-Cano SJ. Molecular and kinetic features of transitional cell carcinomas of the bladder: biological and clinical implications. Virchows Arch 2001; 43828997.

2. Lipponen PK, Aaltoma S. Apoptosis in bladder cancer as related to standard prognostic factors and prognosis. J Pathol 1994; 173: 3339.

3. Moch H, Sauter G, Mihatsch MJ, Gudat F, Epper R, Waldman FM. P53 but not erbB-2 expression is associated with rapid tumor proliferation in urinary bladder cancer. Hum Pathol 1994; 25: 134651

4. McKnight JJ, Gray SB, O'Kane HF, Johnston SR, Williamson KE. Apoptosis and chemotherapy for bladder cancer. J Urol 2005; 173: 68390 .

5. Tabassum A, Khwaja F, Djakiew D. The p75 (NTR) tumor suppressor induces caspase-mediated apoptosis on bladder tumor cells. Int $J$ Cancer 2003; 105: 47.

6. Reed JC. Mechanisms of apoptosis. Am J Pathol 2000; 157: 141530

7. Cryns V, Yuan J. Proteases to die for. Genes Dev 1998; 12: 155170.

8. Thornberry N, Lazebnik Y. Caspases: enemies within. Science 1998 281: 13126.

9. Slee EA, Adrain C, Martin SJ. Executioner caspase-3, -6, and -7 perform distinct, non-redundant roles during the demolition phase of apoptosis. J Biol Chem 2001; 276: 73206.

10. Duan WR, Garner DS, Williams SD, et al. Comparison of immunohistochemistry for activated caspase- 3 and cleaved cytokeratin 18 with the TUNEL method for quantitation of apoptosis in histological sections of PC-3 subcutaneous xenografts. J Pathol 2003; 199: 2218.

11. Korkolopoulou P, Konstantinidou AE, Christodoulou P, et al. Apoptosis in bladder carcinomas detected with monoclonal antibody to single-stranded DNA: relation to cell cycle regulators and survival. Urology 2000; 56: 51620. 
12. Sobin LH, Wittekind C. International Union Against Cancer (UICC): TNM Classification of Malignant Tumours. $6^{\text {th }}$ ed. New York: WileyLiss, 2002.

13. Lopez-Beltran A, Sauter G, Gasser T. Pathology and Genetics of Tumours of the Urinary System and Male Genital Organs. Lyon: IARC Press, 2002; Infiltrating urothelial carcinoma.

14. Torhorst $\mathbf{J}$, Bucher $\mathbf{C}$, Kononen $\mathbf{J}$, et al. Tissue microarrays for rapid linking of molecular changes to clinical endpoints. Am J Pathol 2001 159: 224956.

15. Mitra AP, Datar RH, Cote RJ. Molecular staging of bladder cancer. BJU International 2005; 96: 712.

16. Habuchi T, Marberger M, Droller MJ, et al. Prognostic markers for bladder cancer: international consensus panel on bladder tumor markers. Urology 2005; 66(Suppl 6A): 6474

17. Evan GI, Vousden KH. Proliferation, cell cycle and apoptosis in cancer. Nature 2001; 411 : 3428 .

18. Burton PB, Anderson CJ, Corbishly CM. Caspase 3 and p27 as predictors of invasive bladder cancer. $N$ Engl J Med 2000; 343 141820.

19. Juin $\mathrm{P}$, Hunt A, Littlewood $\mathrm{T}$, et al. C-myc functionally cooperates with Bax to induce apoptosis. Mol Cell Biol 2002; 22: 615869.
20. Karam JA, Lotan Y, Karakiewicz PI, et al. Use of combined apoptosis biomarkers for prediction of bladder cancer recurrence and mortality after radical cystectomy. Lancet Oncol 2007; 8: 12836.

21. Enari M, Sakahira H, Yokoyama $H$, et al. A caspase-activated DNAse that degrades DNA during apoptosis, and its inhibitor ICAD. Nature 1998; 391: 4350 .

22. Chowdhury I, Tharakan B, Bhat GK. Current concepts in apoptosis: The physiological suicide program revisited. Cell Mol Biol Lett 2006; 11: 50625 .

23. Esrig D, Elmajian D, Groshen S, et al. Accumulation of nuclear p53 and tumor progression in bladder cancer. $N$ Engl $J$ Med 1994; 331: 125964.

24. Schmitz-Dräger BJ, Goebell PJ, Ebert $\mathrm{T}$, et al. $\mathrm{P} 53$ immunohistochemistry as a prognosis marker in bladder cancer. Playground for urology scientists? Eur Urol 2000; 38: 6919.

25. Glick SH, Howell LP, Devere White RW. Relationship of p53 and bcl2 to prognosis in muscle-invasive transitional cell carcinoma of the bladder. J Urol 1996; 155: 17547.

26. Smith WL, Marnett LJ, DeWitt DL. Prostanglandin and thromboxane biosynthesis. Pharmacol Ther 1991; 49: 15379.

27. Shirahama T, Arima J, Akiba S. Relation between cyclooxygenase-2 expression and tumor invasiveness and patient survival in transitional cell carcinoma of the urinary bladder. Cancer 2001; 92: 18893. 